\title{
Homozygous NOTCH3 p.R587C mutation in Chinese patients with CADASIL: a case report
}

\author{
Ruojie He${ }^{1}$, Huan Li ${ }^{1}$, Yiming Sun², Menglong Chen ${ }^{3}$, Liang Wang ${ }^{1}$, Yuling Zhu ${ }^{1}$ and Cheng Zhang ${ }^{1 *}$
}

\begin{abstract}
Background: Cerebral autosomal dominant arteriopathy with subcortical infarcts and leukoencephalopathy (CADASIL) is an inherited small vessel disease caused by mutations in NOTCH3 gene with remarkable phenotypic heterogeneity. Cases of CADASIL associated with homozygous NOTCH3 mutations are rare and subsequently understudied. In this study, we investigate the genetic and phenotypic features within patients of CADASIL with homozygous NOTCH3 mutations.

Case presentation: We recruited two affected individuals with CADASIL from a mainland Chinese family. The proband (Patient 1), a 60-year-old male, presented with slow progressive gait instability, severe cognitive impairment, and emotional disorder for more than 2 years with a history of ischemic stroke and hypertension. His younger brother (Patient 2) presented with apparent gait difficulties, dysarthria as well as cognitive decline at 59 years old. Brain magnetic resonance imaging (MRI) showed diffused white matter lesions involving bilateral periventricular white matter, semioval center region, and anterior temporal lobes. Molecular genetic testing identified a homozygous variant, c.1759C > T (p.R587C), in NOTCH3 gene in both patients. Pathological analysis revealed granular osmiophilic material (GOM) deposits in small arterial walls of skin from the proband. The diagnosis of CADASIL was confirmed.

Conclusions: Our cases of CADASIL with homozygous mutation c.1759C > T (p.R587C) in NOTCH3 share similar manifestation to the patients with heterozygous same mutation reported previously. Other than genetic factors, vascular risk factors or environmental factors might contribute to the phenotypic variation of CADASIL.
\end{abstract}

Keywords: CADASIL, NOTCH3 mutation, Homozygous, Phenotypic variation

\section{Background}

Cerebral autosomal dominant arteriopathy with subcortical infarcts and leukoencephalopathy (CADASIL) (OMIM: 125310) is an autosomal dominant small vessel disease predominantly caused by mutations in $\mathrm{NOTCH} 3$ gene (HGNC ID: 7883), which encodes internal, external

\footnotetext{
* Correspondence: chengzhang100@foxmail.com

${ }^{1}$ Department of Neurology, The First Affiliated Hospital, Sun Yat-sen University; Guangdong Provincial Key Laboratory of Diagnosis and Treatment of Major Neurological Diseases, National Key Clinical Department and Key Discipline of Neurology, No.58 Zhongshan Road 2, Guangzhou 510080, China Full list of author information is available at the end of the article
}

and transmembrane domains of $\mathrm{NOTCH} 3$ protein. The extracellular domain consists of 34 epidermal growth factor-like repeats (EGFR), each including 6 cysteine residues. Most identified mutations localize in $\mathrm{NOTCH} 3$ result in an odd number of cysteine residues within a given EGFR. The clinical presentations include recurrent subcortical ischemic events, cognitive impairment or vascular dementia, migraine, psychiatric disorders as well as diffuse white matter lesions and lacunar infarcts in brain magnetic resonance imaging (MRI) [1, 2]. Systemically, patients develop pathognomonic granular osmiophilic material (GOM) deposits in the vessel walls, 
which could be detected in skin biopsies using electron microscopy [3].

Since the first identification of $\mathrm{NOTCH} 3$ mutations in 1996 [4], over 200 mutations have been identified [5]. However, few cases homozygous for a NOTCH3 mutation have been reported to date [6-12], and the specific phenotype-genotype spectrum of patients with homozygous NOTCH3 mutations has not been delineated. In addition, it remains to be determined whether the homozygosity of NOTCH3 mutation is responsible for more severe phenotype in patients of CADASIL, and existing reports offer contradictory observations $[8,10]$.

Here, we report the first patients of CADASIL from a Chinese family with homozygous NOTCH3 mutation c.1759C > T (p.R587C), and pathological analysis confirm GOM deposits in the vessel walls in proband patient.

\section{Case presentation \\ Patient 1}

The proband, a 60-year-old man, suffered from progressive gait instability particularly when walking up and down stairs, cognitive decline, and emotional disorder for more than 2 years. At the age of 47 years, he experienced an ischemic stroke and had mild disability in his left limbs. In the following 10 years, left side hemiparesis did not progress. There was no history of migraine and dizziness. He had hypertension for more than two decades and blood pressure was not well controlled. Neurological examination revealed impaired higher cortical function, gait ataxia, positive Romberg's sign, the presence of bilateral Rossolimo's signs, and palm-chin reflexes. Decreased muscle strength and brisk tendon reflexes were found on the bilateral lower limbs. The neuropsychological evaluation showed considerable cognitive decline including impairments of memory and language ability, with mood disturbances manifesting as both apathy and depression. His performance on the mini-mental status examination (MMSE) showed a moderate cognitive impairment (a score of 20 out of 30). However, he scored 14 out of 30 on the Montreal Cognitive Assessment (MOCA), indicating severe cognitive impairment. Neck vessel doppler sonography revealed left carotid atherosclerosis and the echocardiography evaluation showed hypertrophy of the ventricular septum and enlargement of the left atrium. Brain MRI on T2-weighted and fluid attenuation inversion recovery (FLAIR) imaging revealed diffused white matter hyperintensities mostly involving the bilateral periventricular white matter, semioval center, and left anterior temporal lobe in association with multiple lacunar infarcts predominantly located in subcortical white matter, brainstem, and basal ganglia (Fig. 1a-c, e-g). The small lacunar infarcts could be readily observed on T1- weighted imaging with decreased signal intensity (Fig. 1d). Cerebral magnetic resonance angiography presented stenosis of the intracranial arteries with cerebral arteriosclerosis (Fig. 1h). Based on clinical and neuroimaging findings, he was clinically suspected of having the diagnosis of CADASIL and underwent genetic and pathological analyses.

Written informed consent was obtained from the patients and the study was approved by the Institutional Review Board of The First Affiliated Hospital of Sun Yat-sen University. The direct sequencing of the NOTCH3 gene identified the homozygous c. $1759 \mathrm{C}>\mathrm{T}$ (p.R587C) missense mutation in exon 11 (Fig. 2), which was identified in dbSNP (rs754554486) and Human Gene Mutation Database (HGMD) (CM061879) with a gnomAD allele frequency of $3.616 \mathrm{e}-5$. This change was predicted as damaging variant based on analysis using PolyPhen-2 software. Furthermore, the patient underwent skin biopsy on his right lower limb and electron microscopic analysis revealed GOM deposits between vascular smooth muscle cells (SMCs) and the basement membrane, and occasionally within SMCs in small arterial walls (Fig. 3).

\section{Patient 2}

The younger brother of the proband was a 59-year-old man presenting with apparent gait difficulties and clumsiness of movement from the age of 57 . He gradually developed slurred speech and mild weakness of the bilateral upper limbs, and had distinct difficulty in completing routine work. He had a transient ischemic attack (TIA) at the age of 50 and recovered. There was also no history of migraine. No vascular risk factors were found. The neurological examination revealed gait ataxia, dysarthria, reduced mild power on bilateral upper limbs as well as brisk deep tendon reflexes in all limbs. The bilateral Rossolimo's signs and right palm-chin reflex were positive. Neuropsychological examination showed impairment of linguistic and calculative functions and mood disturbance like apathy. Brain MRI findings demonstrated confluent and symmetrical distribution of white matter lesions in bilateral periventricular white matter, semioval center region, and anterior temporal lobes on T2-weighted and FLAIR imaging. Multiple lacunar infarcts were detected predominantly in basal ganglia, thalamus, brainstem, and subcortical white matter (Fig. 1i-l). His MMSE score was 23 out of 30, indicating a mild cognitive impairment. The same homozygous NOTCH3 c.1759C > T (p.R587C) mutation was detected (Fig. 2).

The proband's father had a history of ischemic stroke and died at the age of 50, and the proband's mother died at the age of 69 because of heart disease. Unfortunately, no further clinical information from either individual was available. The proband's son, a 34-year-old man, 

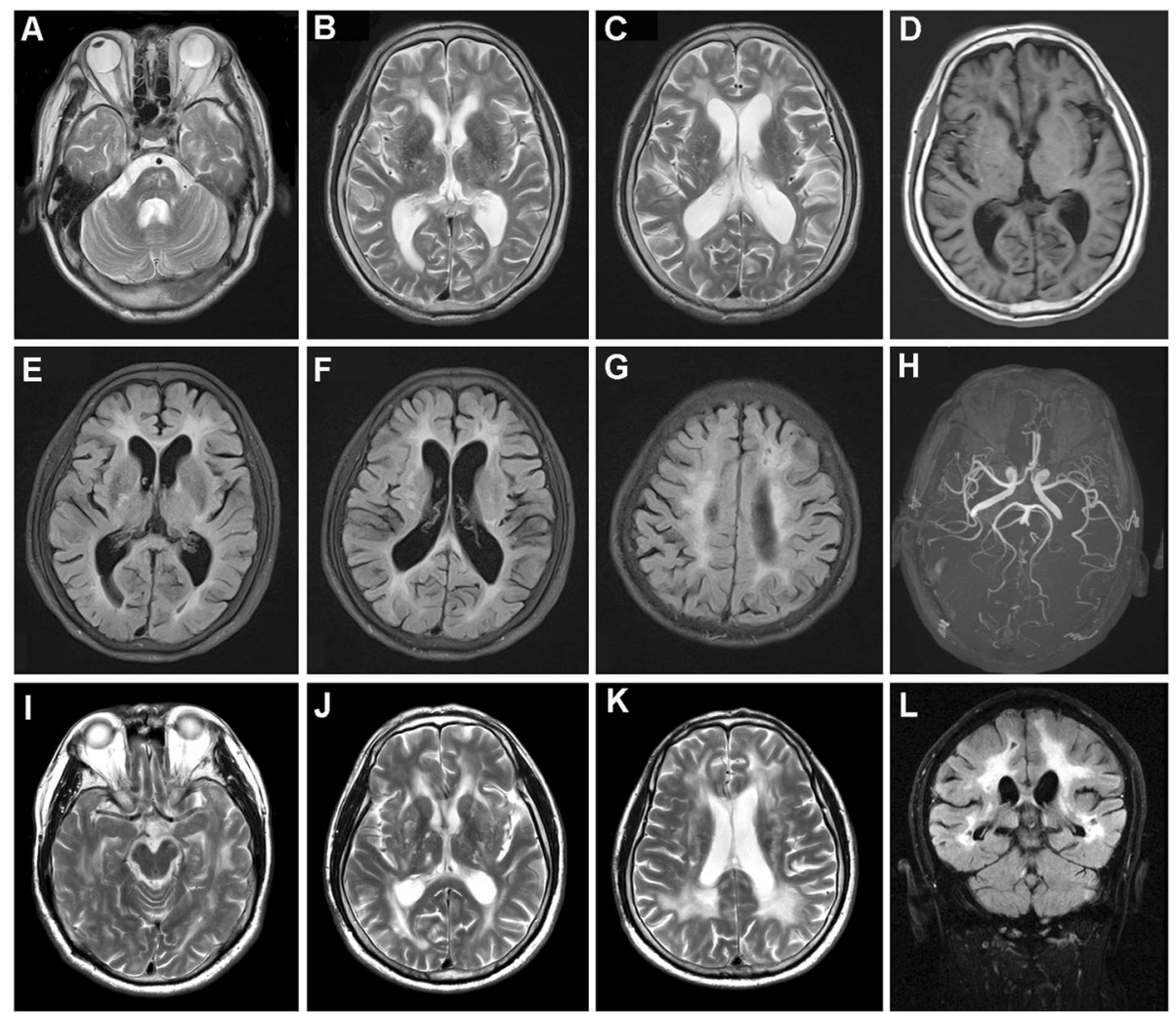

Fig. 1 Brain MRI of the patient. Brain MRI of the patient 1 (a-h) and patient 2 (i-I) in the family with CADASIL showed diffused white matter hyperintensities as well as lacunar infarcts on T1-weighted, T2-weighted and FLAIR imaging

was clinically asymptomatic and his neuroimaging finding was normal. Nevertheless, the heterozygous NOTCH3 c.1759C > T (p.R587C) mutation was detected (Fig. 2).

\section{Discussion and conclusions}

We reported the homozygous missense c. $1759 \mathrm{C}>\mathrm{T}$ (p.R587C) mutation in NOTCH3 gene discovered in two brothers from a mainland Chinese family with CADASIL confirmed pathologically. Clinically, both patients in this study presented with ischemic stroke, gait instability, cognitive impairment, and psychiatric disorders which were highly compatible with the phenotypic spectrum of CADASIL in previous studies from both Chinese and other ethnic backgrounds [13-16]. The confluent white matter hyperintensities as well as multiple lacunar infarcts shown on brain MRI scanning of our patients were in accordance with typical neuroimaging features of CADASIL. None of the patients in present study reported a history of migraine with or without aura, which is consistent with a relatively low frequency of migraine in patients of CADASIL from Asian countries [16, 17]. The diagnosis of CADASIL was genetically confirmed by the detection of a homozygous mutation in exon 11 of NOTCH3 gene, which was predicted to cause a substitution of arginine with cysteine (p.R587C) in the encoded receptor. The ultrastructural examination of dermal arteries performed via skin biopsy from the proband showed pathognomonic GOM deposits around irregularly shaped SMCs. In addition, the proband's son, a heterozygous carrier of NOTCH3 c.1759C > T (p.R587C) mutation, was asymptomatic likely due to his young age. Though clinical and genetic assessment were unavailable from the proband's parents, we posited that they were likely to be carrying the heterozygous NOTCH3 c. $1759 \mathrm{C}>\mathrm{T}$ (p.R587C) mutation due to the homozygosity in the proband and his younger brother.

To our knowledge, the homozygous NOTCH3 c.1759C > T (p.R587C) mutation has not been reported before, though the heterozygous cases were discussed in previous studies $[15,18,19]$. Whether homozygous NOTCH3 mutations are associated with much more severe phenotypes in patients with CADASIL remains to be controversial. Some patients with homozygous NOTCH3 mutations exhibit more aggressive clinical or pathological manifestations when compared to their heterozygous family members or age-matched patients with heterozygous mutations $[7,9,10]$, whereas others exhibit similar phenotypes with heterozygous patients $[6,8]$. In 

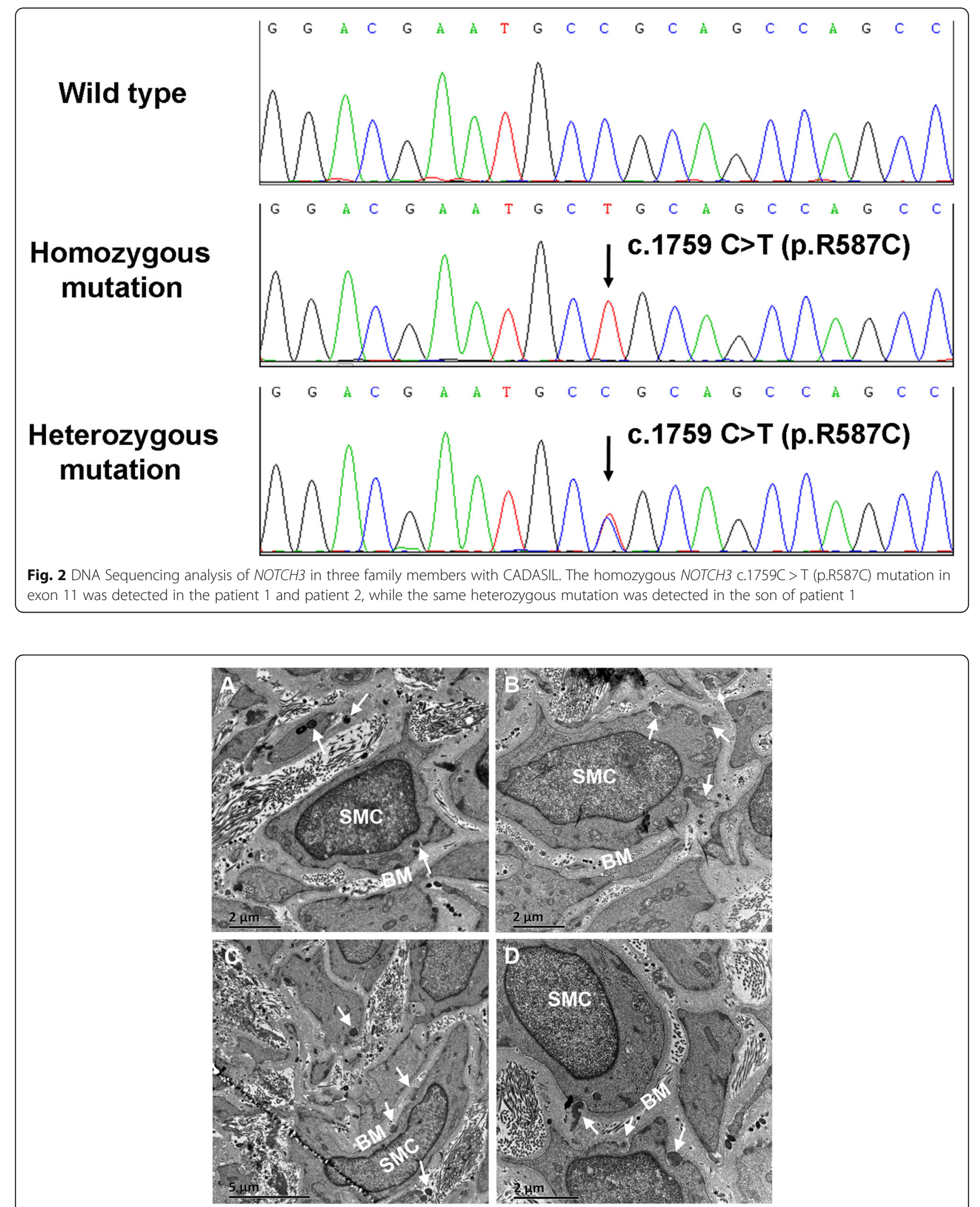

Fig. 3 Ultrastructural analysis using Transmission electron microscopy (TEM) of small dermal arteries from skin biopsy of the proband. GOM deposits (white arrows) showing different shapes were mainly located between vascular SMC and the basement membrane (BM). $\mathbf{a}, \mathbf{b}$ TEM magnification $\times 8000$. c TEM magnification $\times 5000$. $\mathbf{d}$ TEM magnification $\times 10,000$ 
a study from Taiwan, a proband with homozygous c.1630C > T (p.R544C) mutation in NOTCH3 gene presented with a later age of onset and mildly increased clinical severity compared to her heterozygous sister [9]. In another report, a 65-year-old patient with homozygous NOTCH3 c.1732C > T (p.R578C) mutation had parallel phenotypes to those who harbored heterozygous one [6]. In a recent study, a case of CADASIL from Thailand with a homozygous c. $1672 \mathrm{C}>\mathrm{T}$ mutation in $\mathrm{NOTCH} 3$ gene presented with the largest number of cerebral microbleeds ever recorded, probably caused by a homozygous state and uncontrolled vascular risk factors [20]. In present study, clinical manifestations including ischemic stroke, motor disability, cognitive impairment, mood disturbance as well as lack of migraine in our patients with homozygous NOTCH3 c.1759C > T (p.R587C) mutation were accordant with those described in a Korean patient who was heterozygous for the same mutation. Moreover, the diffused white matter lesions on brain MRI examination and GOM deposits in dermal arterioles were comparable between our patients and the Korean patient [18]. These observations suggest that the homozygosity of NOTCH3 c.1759C > T (p.R587C) mutation are probably not associated with more severe phenotypes of CADASIL.

Previous studies indicated that the identical NOTCH3 gene mutation may result in considerable phenotypic variability across unrelated patients, and even members within the same family, suggesting that additional genetic factors, environmental cues or other vascular risk factors are likely to influence the disease progression [9]. Intriguingly, the proband patient and his younger brother in this study displayed mild variation on clinical phenotype though carrying the identical NOTCH3 gene mutation and from the same family. The onset of age for the first ischemic stroke was earlier in the proband when compared to his younger brother. Accordingly, the proband had vascular risk factors like hypertension and left carotid atherosclerosis whereas his younger brother had no vascular risk factors, which indicated the existence of modifying factors other than sole NOTCH3 gene mutation on disease progression and phenotypic variability, as described in previous reports.

In conclusion, we present two cases of CADASIL from a mainland Chinese family with homozygous NOTCH3 c.1759C > T (p.R587C) mutation, one of them is confirmed pathologically. The clinical presentations of our patients with homozygous NOTCH3 c.1759C $>\mathrm{T}$ (p.R587C) mutation are in accordance with that of patients with heterozygous the same mutation described before. Vascular risk factors, environmental factors, and additional unknown genetic factors might contribute to phenotypical heterogeneity of CADASIL.

\section{Abbreviations}

CADASIL: Cerebral autosomal dominant arteriopathy with subcortical infarcts and leukoencephalopathy; EGFR: Epidermal growth factor-like repeats; FLAIR: Fluid attenuation inversion recovery; GOM: Granular osmiophilic material; HGMD: Human Gene Mutation Database; MMSE: Mini-mental status examination; MOCA: Montreal Cognitive Assessment; MRI: Magnetic resonance imaging; SMCs: Vascular smooth muscle cells; TIA: Transient ischemic attack

\section{Acknowledgments}

Not applicable.

\section{Authors' contributions}

$\mathrm{RH}$ contributed to the acquisition of data, analysis and interpretation of data, and wrote the manuscript. $\mathrm{HL}$ and YS contributed to the analysis and interpretation of data and the manuscript editing. MC and LW contributed to the genetic analysis. CZ and $Y Z$ revised the manuscript critically. All authors read and approved the final manuscript.

\section{Funding}

This work was supported by grants from the National Natural Science Foundation of China (no.81471280 and 81771359) which were used to support the design of the study and data collection; the Young Scientist Fund of National Natural Science Foundation of China (no. 81601087) which was used to support the molecular genetic testing; the Guangzhou Science and Technology Program (no.1561000153) which was used to support the data analysis and interpretation; the Southern China International Cooperation Base for Early Intervention and Functional Rehabilitation of Neurological Diseases (no. 2015B050501003) which was used to support the data analysis and interpretation; the Guangdong Provincial Engineering Center for Major Neurological Disease Treatment, Guangdong Provincial Translational Medicine Innovation Platform for Diagnosis and Treatment of Major Neurological Disease, and Guangdong Provincial Clinical Research Center for Neurological Diseases, which were used to support in writing the manuscript.

Availability of data and materials

The authors declare that all the data are contained within the manuscript.

Ethics approval and consent to participate

This study was approved by the Institutional Review Board of the First Affiliated Hospital of Sun Yat-sen University (NO. [2016]87).

\section{Consent for publication}

The written informed consent was obtained from the patients. The consent was for publication of the patients' of potentially identifying personal and medical information including any associated images.

\section{Competing interests}

The authors declare that they have no competing interests. The study was supported by grants from the National Natural Science Foundation of China (no.81471280 and 81771359); the Young Scientist Fund of National Natural Science Foundation of China (no. 81601087); the Guangzhou Science and Technology Program (no.1561000153); the Southern China International Cooperation Base for Early Intervention and Functional Rehabilitation of Neurological Diseases (no. 2015B050501003); the Guangdong Provincial Engineering Center for Major Neurological Disease Treatment, Guangdong Provincial Translational Medicine Innovation Platform for Diagnosis and Treatment of Major Neurological Disease, and Guangdong Provincial Clinical Research Center for Neurological Diseases.

\section{Author details}

${ }^{1}$ Department of Neurology, The First Affiliated Hospital, Sun Yat-sen University; Guangdong Provincial Key Laboratory of Diagnosis and Treatment of Major Neurological Diseases, National Key Clinical Department and Key Discipline of Neurology, No.58 Zhongshan Road 2, Guangzhou 510080, China. ${ }^{2}$ Department of Health Care, The First Affiliated Hospital, Sun Yat-sen University, No.58 Zhongshan Road 2, Guangzhou 510080, China.

${ }^{3}$ Department of Neurology, The First Affiliated Hospital, Jinan University, 613 W.Huangpu Avenue, Guangzhou 510630, China. 
Received: 31 August 2019 Accepted: 26 February 2020

Published online: 02 March 2020

\section{References}

1. Peters N, Holtmannspotter M, Opherk C, Gschwendtner A, Herzog J, Samann $P$, et al. Brain volume changes in Cadasil: a serial Mri study in pure subcortical ischemic vascular disease. Neurology. 2006:66(10):1517-22. https://doi.org/10.1212/01.wnl.0000216271.96364.50.

2. Jouvent E, Mangin JF, Porcher R, Viswanathan A, O'Sullivan M, Guichard JP, et al. Cortical changes in cerebral small vessel diseases: a 3D Mri study of cortical morphology in Cadasil. Brain. 2008;131(Pt 8):2201-8. https://doi.org/ 10.1093/brain/awn129.

3. Chabriat $H$, Joutel A, Dichgans M, Tournier-Lasserve E, Bousser MG. Cadasil. Lancet Neurol. 2009;8(7):643-53. https://doi.org/10.1016/S14744422(09)70127-9.

4. Joutel A, Corpechot C, Ducros A, Vahedi K, Chabriat H, Mouton P, et al. Notch3 mutations in Cadasil, a hereditary adult-onset condition causing stroke and dementia. Nature. 1996;383(6602):707-10. https://doi.org/10. 1038/383707a0

5. Tikka S, Baumann M, Siitonen M, Pasanen P, Poyhonen M, Myllykangas L, et al. Cadasil and Carasil. Brain Pathol. 2014;24(5):525-44. https://doi.org/10 1111/bpa.12181.

6. Liem MK, Lesnik OS, Vollebregt MJ, Middelkoop HA, van der Grond J, Helderman-van DEA. Homozygosity for a Notch3 mutation in a 65-year-old Cadasil patient with mild symptoms: a family report. J Neurol. 2008;255(12): 1978-80. https://doi.org/10.1007/s00415-009-0036-x.

7. Tuominen S, Juvonen V, Amberla K, Jolma T, Rinne JO, Tuisku S, et al. Phenotype of a homozygous Cadasil patient in comparison to 9 agematched heterozygous patients with the same R133C Notch3 mutation. Stroke. 2001:32(8):1767-74. https://doi.org/10.1161/01.str.32.8.1767.

8. Ragno M, Pianese L, Morroni M, Cacchio G, Manca A, Di Marzio F, et al. "Cadasil coma" in an Italian homozygous Cadasil patient: comparison with clinical and Mri findings in age-matched heterozygous patients with the same G528C Notch3 mutation. Neurol Sci. 2013;34(11):1947-53. https://doi. org/10.1007/s10072-013-1418-5

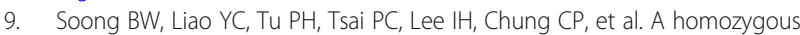
Notch3 mutation p.R544C and a heterozygous Trex1 variant P.C99Mfsx3 in a family with hereditary small vessel disease of the brain. J Chin Med Assoc. 2013;76(6):319-24. https://doi.org/10.1016/j.jcma.2013.03.002.

10. Vinciguerra C, Rufa A, Bianchi S, Sperduto A, De Santis M, Malandrini A, et al. Homozygosity and severity of phenotypic presentation in a Cadasil family. Neurol Sci. 2014;35(1):91-3. https://doi.org/10.1007/s10072-013-1580-9.

11. Abou AH, Qadi N, Al-Hamed MH, Meyer BF, Bohlega S. Phenotypic comparison of individuals with homozygous or heterozygous mutation of Notch3 in a large Cadasil family. J Neurol Sci. 2016;367:239-43. https://doi. org/10.1016/j.jns.2016.05.061.

12. Mukai M, Mizuta I, Ueda A, Nakashima D, Kushimura $Y$, Noto Yl, et al. A Japanese Cadasil Patient with Homozygous Notch3 P.Arg544Cys Mutation Confirmed Pathologically. J Neurol Sci. 2018;394:38-40. https://doi.org/10. 1016/j.jns.2018.08.029

13. Kim Y, Choi EJ, Choi CG, Kim G, Choi JH, Yoo HW, et al. Characteristics of Cadasil in Korea: a novel cysteine-sparing Notch3 mutation. Neurology. 2006;66(10):1511-6. https://doi.org/10.1212/01.wnl.0000216259.99811.50.

14. Bianchi S, Zicari E, Carluccio A, Di Donato I, Pescini F, Nannucci S, et al. Cadasil in Central Italy: a retrospective clinical and genetic study in 229 patients. J Neurol. 2015;262(1):134-41. https://doi.org/10.1007/s00415-0147533-2.

15. Liao YC, Hsiao CT, Fuh JL, Chern CM, Lee WJ, Guo YC, et al. Characterization of Cadasil among the Han Chinese in Taiwan: distinct genotypic and phenotypic profiles. PLoS One. 2015;10(8):e136501. https://doi.org/10.1371/ journal.pone.0136501.

16. Liu X, Zuo Y, Sun W, Zhang W, Lv H, Huang Y, et al. The genetic Spectrum and the evaluation of Cadasil screening scale in Chinese patients with Notch3 mutations. J Neurol Sci. 2015;354(1-2):63-9. https://doi.org/10.1016/ j.jns.2015.04.047.

17. Chen S, Ni W, Yin X, Liu H, Lu C, Zheng Q, et al. Clinical features and mutation Spectrum in Chinese patients with Cadasil: a multicenter retrospective study. CNS Neurosci Ther. 2017;23(9):707-16. https://doi.org/ $10.1111 /$ cns.12719.
18. Kim Y, Kim JS, Kim G, No YJ, Yoo HW. Two novel mutations of the Notch3 gene in Korean patients with Cadasil. Mutat Res. 2006;593(1-2):116-20. https://doi.org/10.1016/j.mrfmmm.2005.06.031.

19. He D, Chen D, Li X, Hu Z, Yu Z, Wang W, et al. The comparisons of phenotype and genotype between Cadasil and Cadasil-like patients and population-specific evaluation of Cadasil scale in China. J Headache Pain. 2016;17:55. https://doi.org/10.1186/s10194-016-0646-5.

20. Anamnart C, Songsaeng D, Chanprasert S. A large number of cerebral microbleeds in Cadasil patients presenting with recurrent seizures: a case report. BMC Neurol. 2019;19(1):106. https://doi.org/10.1186/s12883-019-1342-2.

\section{Publisher's Note}

Springer Nature remains neutral with regard to jurisdictional claims in published maps and institutional affiliations.
Ready to submit your research? Choose BMC and benefit from:

- fast, convenient online submission

- thorough peer review by experienced researchers in your field

- rapid publication on acceptance

- support for research data, including large and complex data types

- gold Open Access which fosters wider collaboration and increased citations

- maximum visibility for your research: over $100 \mathrm{M}$ website views per year

At BMC, research is always in progress.

Learn more biomedcentral.com/submissions 